\title{
Abdominal organ position variation in children during image-guided radiotherapy
}

Sophie C. Huijskens, Irma W. E. M. van Dijk, Jorrit Visser, Brian V. Balgobind, D. te Lindert, Coen R. N. Rasch, Tanja Alderliesten and Arjan Bel

\begin{abstract}
Background: Interfractional organ position variation might differ for abdominal organs and this could have consequences for defining safety margins. Therefore, the purpose of this study is to quantify interfractional position variations of abdominal organs in children in order to investigate possible correlations between abdominal organs and determine whether position variation is location-dependent.

Methods: For 20 children (2.2-17.8 years), we retrospectively analyzed 113 CBCTs acquired during the treatment course, which were registered to the reference $C T$ to assess interfractional position variation of the liver, spleen, kidneys, and both diaphragm domes. Organ position variation was assessed in three orthogonal directions and relative to the bony anatomy. Diaphragm dome position variation was assessed in the cranial-caudal (CC) direction only. We investigated possible correlations between position variations of the organs (Spearman's correlation test, $\rho)$, and tested if organ position variations in the CC direction are related to the diaphragm dome position variations (linear regression analysis, $\mathrm{R}^{2}$ ) (both tests: significance level $p<0.05$ ). Differences of variations of systematic $(\Sigma)$ and random errors $(\sigma)$ between organs were tested (Bonferroni significance level $p<0.004$ ).
\end{abstract}

Results: In all directions, correlations between liver and spleen position variations, and between right and left kidney position variations were weak $(\rho \leq 0.43)$. In the CC direction, the position variations of the right and left diaphragm domes were significantly, and stronger, correlated with position variations of the liver $\left(R^{2}=0.55\right)$ and spleen $\left(R^{2}=0.63\right)$, respectively, compared to the right $\left(R^{2}=0.00\right)$ and left kidney $\left(R^{2}=0.25\right)$. Differences in $\Sigma$ and $\sigma$ between all organs were small and insignificant.

Conclusions: No (strong) correlations between interfractional position variations of abdominal organs in children were observed. From present results, we concluded that diaphragm dome position variations could be more representative for superiorly located abdominal (liver, spleen) organ position variations than for inferiorly located (kidneys) organ position variations. Differences of systematic and random errors between abdominal organs were small, suggesting that for margin definitions, there was insufficient evidence of a dependence of organ position variation on anatomical location.

Keywords: Interfractional organ position variation, Abdominal organ motion, Pediatric RT, IGRT

\section{Background}

Continuous developments in pediatric cancer treatment using multimodality strategies, including surgery, chemotherapy, and radiotherapy have led to increasing numbers of childhood cancer survivors [1]. Inevitably, the occurrence of treatment associated adverse events has also increased. Treatments including radiotherapy significantly contribute to the risk of developing adverse events.

\footnotetext{
* Correspondence: s.c.huijskens@amc.uva.nl

Amsterdam UMC, University of Amsterdam, Department of Radiation

Oncology, Cancer Center Amsterdam, Meibergdreef 9, Amsterdam, The Netherlands
}

Children are treated with abdominal and thoracic radiotherapy for a wide range of primary cancer diagnoses, including Wilms' tumor, neuroblastoma, and Ewing sarcoma. Moreover, treatment of the craniospinal axis and lung metastasis involve irradiation of the abdominal and thoracic region. The anatomical locations of these tumors and adjacent organs at risk (OARs) vary; target volumes can be in very close proximity to the lungs, diaphragm, liver, spleen, and kidneys. As a result, healthy tissues and OARs are unavoidably exposed to radiation when irradiating the tumor $[2,3]$. Although adequate tumor dose coverage is the

(c) The Author(s). 2018 Open Access This article is distributed under the terms of the Creative Commons Attribution 4.0 International License (http://creativecommons.org/licenses/by/4.0/), which permits unrestricted use, distribution, and reproduction in any medium, provided you give appropriate credit to the original author(s) and the source, provide a link to the Creative Commons license, and indicate if changes were made. The Creative Commons Public Domain Dedication waiver (http://creativecommons.org/publicdomain/zero/1.0/) applies to the data made available in this article, unless otherwise stated. 
primary goal in radiotherapy, sparing the vital and long-term functions of adjacent organs is also of great concern. Especially in children, who have a relative long life expectancy when surviving cancer, organs are still growing and have low tolerance to radiation $[4,5]$. To ensure adequate tumor dose coverage while minimizing radiation dose to surrounding healthy tissues, knowledge about the extent of target and organ motion, particularly present in the abdominal and thoracic area, is needed. Thus, quantifying the motion of vital and sensitive organs such as the liver, spleen, and kidneys is essential.

These abdominal organs move with every breathing cycle (intrafraction motion) and from day-to-day (interfraction motion). Intra- and interfractional motion of the tumor and OARs are incorporated by expanding the clinical target volume and OARs volumes to the planning target volume (PTV) and planning risk volumes (PRVs), respectively [6]. In adults, many studies have quantified motion of various organs, enabling to define accurate margins for PTVs and PRVs. Despite the increasing number of publications on pediatric organ motion [7-14], data is still limited and no consensus has been reached in pediatric radiotherapy to define PTV or PRV margins for abdominal tumors or OARs. Therefore, PTV margins for children are currently pragmatically based on available adult data and PRV margins are often not used in pediatric radiotherapy. Due to different anatomical locations (e.g., right vs. left side of the abdomen, (retro)peritoneum, adjacent to the vertebrae), or abdominal processes (e.g., intestinal peristaltic or air pockets), abdominal organ motion might be locationdependent, as was discussed before in Van Dijk et al. [14]. This could lead to differences in PTV and PRV margins depending on the anatomical location.

The most commonly used PTV margin recipe is from van Herk et al. $(2.5 \Sigma+0.7 \sigma)$, where the systematic $(\Sigma)$ and random $(\sigma)$ component are based on quadratically adding the systematic/random errors that occur during treatment $\left(\sqrt{\Sigma_{\text {inter }}^{2}+\Sigma_{\text {intra }}^{2}}\right.$ and $\left.\sqrt{\sigma_{\text {inter }}^{2}+\sigma_{\text {intra }}^{2}}\right)$ [15]. Previous studies mainly reported on intrafractional organ motion, focusing on respiratory-induced abdominal organ motion through various phases of the breathing cycle as measured on a single four-dimensional computed tomography $(4 \mathrm{DCT})[9,11,16]$ or $4 \mathrm{D}$ magnetic resonance imaging (4DMRI) [12, 17]. Although organ motion seems to be more prone to respiratory motion than to day-to-day position variations, Guerreiro et al. showed that in a homogenous group of 15 children, interfractional abdominal organ motion was larger than intrafraction motion $\left(\Sigma_{\text {inter }}\right.$ and $\sigma_{\text {inter }}>\Sigma_{\text {intra }}$ and $\left.\sigma_{\text {intra }}\right)$ [16]. In addition, Huijskens et al. showed that for respiratory-induced diaphragm motion in children the systematic error was found to be smaller than the random error $\left(\sum_{\text {intra }}<\sigma_{\text {intra }}\right)$ [8]. This seems to indicate that the systematic component of the
PTV and PRV margins is predominated by the day-to-day systematic (i.e., interfractional) variations $\left(\Sigma_{\text {inter }}\right)$. Moreover, van Herk's margin recipe shows that the systematic component weighs more than the random component [15]. Therefore, quantification and a comprehensive understanding of interfractional abdominal organ motion is essential for high-accuracy image-guided radiotherapy.

Most studies on abdominal organ motion have focused only on the quantification of the interfractional component $[7,10,16,18]$, without investigating location-dependency, or possible correlations between organ position variations. Whenever possible, resection of a tumor takes place before radiation treatment and usually surgical clips are placed to localize the remaining tumor bed. If not, an anatomical structure close to the target could function as a surrogate for localization and position variation. However, such a strategy will only be successful when there is a clear understanding of the correlations between the tumor and the anatomical surrogate. In addition, radiation treatment might also lead in the future towards adaptive strategies in children. However, often, certain organs are not directly visible on daily cone beam CTs (CBCTs), due to artefacts, smaller field of view or, especially in children, low dose imaging protocols. Moreover, markers are rather not placed in children and online evaluation of the positions of organs is thus mostly unfeasible in clinical practice. Here as well, another close anatomical structure might be considered as a surrogate. For instance, when the diaphragm, being very well visible on CBCT images, is used as a surrogate for the assessment of abdominal organ position. Some adult studies have shown reliable correlations of the diaphragm with abdominal organs [19-22], while other studies show weak correlations [20,23-25]. This is mostly depending on the tumor site and therefore, outcomes cannot be generalized for adults. For children, correlations between the diaphragm and abdominal organs has not been extensively studied. It is therefore crucial to have a clear understanding of the correlation between the tumor or organ and a particular surrogate.

Therefore, the aim of this study was to increase the insight on interfractional position variation of abdominal organs in children. We investigated possible correlations between abdominal organs and determined whether position variation is location-dependent. Additionally, we investigated whether diaphragm position variation could be a surrogate for abdominal organ position variation, by analyzing the right and left diaphragm domes separately.

\section{Methods}

\section{Patient population}

For this retrospective study, we included 20 patients younger than 18 years, treated for various tumors at our radiation oncology department between December 2010 and 
Table 1 Patient characteristics

\begin{tabular}{|c|c|c|c|c|c|c|c|}
\hline No. & Sex & Tumor & Age at diagnosis (years) & Height $(\mathrm{cm})$ & Weight (kg) & No. of CBCTs & RT location \\
\hline 1 & $\mathrm{~F}$ & Sarcoma & 11.5 & 155 & 38 & 5 & Thorax \\
\hline 2 & M & Medulloblastoma & 6.6 & 110 & 18 & 5 & Spinal $\operatorname{cord}^{\mathrm{a}}$ \\
\hline 3 & $\mathrm{~F}$ & Hodgkin lymphoma & 16.5 & 166 & 49 & 5 & abdomen \\
\hline 4 & M & Medulloblastoma & 14.1 & 175 & 36 & 5 & Spinal cord \\
\hline 5 & M & Medulloblastoma & 8.3 & 128 & 25 & 5 & Spinal cord \\
\hline 6 & $\mathrm{~F}$ & Medulloblastoma & 6.7 & 117 & 20 & 2 & Spinal cord \\
\hline 7 & M & Ewing sarcoma & 16.8 & 184 & 62 & 8 & Thorax \\
\hline 8 & M & Medulloblastoma & 6.7 & 129 & 24 & 5 & Spinal cord \\
\hline 9 & M & Spinal metastesis & 2.6 & 90 & 12 & 8 & Thorax \\
\hline 10 & $\mathrm{~F}$ & Medulloblastoma & 7 & 118 & 22 & 6 & Spinal cord \\
\hline 11 & M & Anaplastic glioma & 7.9 & 132 & 31 & 5 & Spinal cord \\
\hline $12^{\mathrm{b}}$ & M & Medulloblastoma & 5.1 & 109 & 17 & 8 & Spinal cord \\
\hline 13 & $\mathrm{~F}$ & Neuroblastoma & 5.3 & 115 & 24 & 6 & Abdomen \\
\hline 14 & M & Sarcoma & 10.9 & 142 & 37 & 5 & Thorax \\
\hline 15 & M & DSRCT & 9.9 & 137 & 26 & 5 & Abdomen \\
\hline 16 & M & Neuroblastoma & 4.7 & 118 & 22 & 6 & Abdomen \\
\hline $17^{\mathrm{b}}$ & M & Medulloblastoma & 4.9 & 105 & 18 & 6 & Spinal cord \\
\hline 18 & M & Ewing sarcoma & 17.9 & 182 & 81 & 7 & Thorax \\
\hline 19 & $\mathrm{~F}$ & Osteosarcoma & 15.1 & 159 & 53 & 5 & Thorax \\
\hline 20 & M & Neuroblastoma & 2.2 & 90 & 15 & 6 & Abdomen \\
\hline
\end{tabular}

Abbreviations: $M$ male, $F$ female, DSRCT desmoplastic small round cell tumor

a Spinal cord was part of craniospinal irradiation

b Patients 12 and 17 were treated under general anesthesia.; this did not influence interfractional organ position variations

September 2017 (Table 1). Patients were included if a pre-treatment $\mathrm{CT}$ scan and multiple $\mathrm{CBCT}$ scans of the abdomen or thorax were available, in which the liver, spleen, kidneys, and right and left diaphragm domes were visible (Fig. 1).

\section{Imaging data}

For each patient, a pre-treatment CT scan $(120 \mathrm{kV}, 2.5$ - or $5 \mathrm{~mm}$ slice thickness) was acquired for planning purposes (LightSpeed RT16; General Electric Company, Waukesha, WI, USA). This scan was considered as the reference CT (refCT) scan and included original organ delineations, as used for clinical practice (Fig. 1). For all patients, CBCT images (1 mm slice thickness, $1 \mathrm{~mm}$ in-plane resolution) were routinely acquired using the $\mathrm{CBCT}$ scanner mounted on the Elekta Synergy linac (Elekta AB, Stockholm, Sweden) as part of the position verification protocol. This yields $\mathrm{CBCT}$ imaging at the first three treatment fractions, followed by daily or weekly CBCT acquisitions, depending on the treatment protocol. To be consistent, we included for all patients the first three CBCTs and thereafter weekly acquired CBCTs. All CBCTs were acquired with $120 \mathrm{kV}$, $10 \mathrm{~mA}$, and 10 or $40 \mathrm{~ms}$ exposure time per projection. The scanning time of the CBCT scan varied between 3560 s, and the degree of circumferential rotation was 200 or
360 degrees. In this study, we retrospectively analyzed the imaging data, including a total of 20 refCTs and 113 CBCTs.

\section{Imaging registration}

Elekta X-ray Volume Imaging software (XVI 3.0; Elekta $\mathrm{AB}$, Stockholm, Sweden) was used for a two-step rigid registration for each organ separately (example shown in Fig. 1). First, a region of interest (ROI) was defined in the refCT, including the 12th thoracic through the 4th lumbar vertebra (from the lowest part of the kidneys up to the diaphragm domes). The $\mathrm{CBCTs}$ were then registered to the refCT using the automatic chamfer match algorithm [26]. Second, this bony anatomy-based match was followed by registration of each organ separately (i.e., liver, spleen, right kidney, left kidney) with a grey value algorithm [26], based on shaped ROIs defined by the delineated organs including (at least 2/3rd of) the whole organ volume. This enabled the assessment of organ position variation smaller than the slice thickness of the acquired refCT. Automatic registration outcomes (translations and rotations) were visually checked (by SCH/DTL) and manually corrected if necessary. Results were corrected for rotations as follows. First, we assessed the center of mass (COM) coordinates for each organ. 

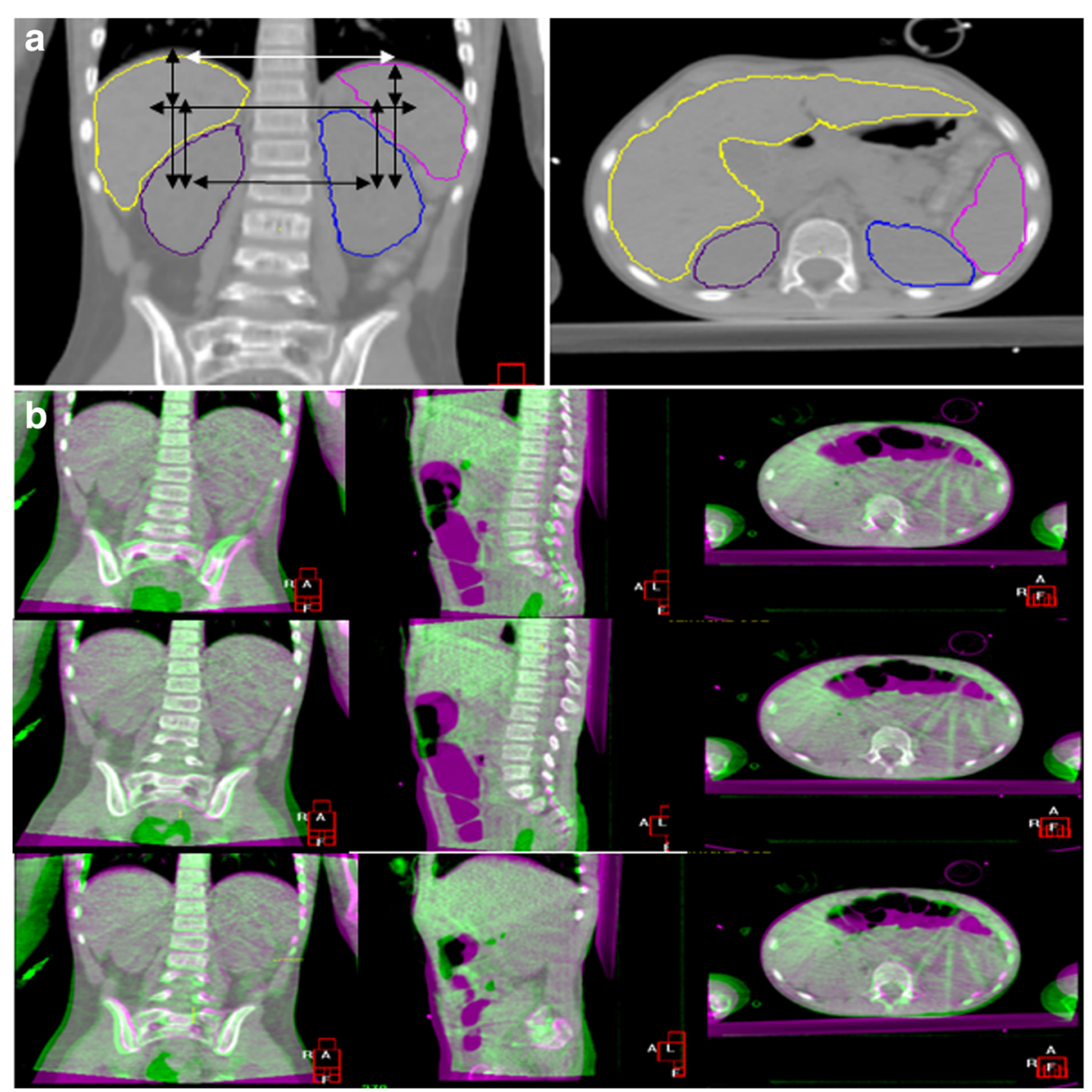

Fig. 1 a Delineated organs (right kidney: purple, left kidney: blue, liver: yellow, spleen: pink) on the reference CT. Diaphragm domes are not delineated. Arrows indicate mutual correlations investigated. $\mathbf{b}$ Example of the two-step rigid registration (from top to bottom): unaligned overlap of reference $C T$ and $\mathrm{CBCT}$, bones aligned, right kidney aligned (note: bones shifted). (Color figure online only)

Then, we equated these coordinates to the refCT to determine the exact magnitude and direction of the interfractional position variation. By calculating the difference of the magnitude and sign of the COM coordinates of each organ on $\mathrm{CBCTs}$ and refCT, registrations resulted in interfractional position variation relative to bony anatomy, expressed as composite vectors in the left-right (LR), cranio-caudal (CC) and anterior-posterior (AP) directions. The + and - signs respectively indicate right/caudal/posterior and left/cranial/anterior directions. For the diaphragm, the bony anatomybased automatic chamfer match was followed by manual registrations of the right- and left-sided diaphragm dome separately in the CC direction only (by SCH/DTL).

\section{Statistical analysis}

For each patient, organ specific mean and standard deviation (SD) of the interfractional position variation relative to the bony anatomy were determined in the three orthogonal directions, and in the CC direction only for the right and left diaphragm domes. Furthermore, over all patients, we estimated per organ the group mean (i.e., mean of the individual means), the group systematic error ( $\sum$; the SD of the individual means of all patients), and the group random error $(\sigma ;$ the root mean square of the individual SDs of all patients).

To evaluate whether organ position variation is locationdependent, we compared contralateral and superiorly and inferiorly located abdominal organs separately (indicated in Fig. 1). Since not all data fitted a normal distribution (tested with the Shapiro-Wilk's test), differences between contralateral organs' systematic and random errors were separately tested (i.e., right diaphragm dome vs. left diaphragm dome, liver vs. spleen, right kidney vs. left kidney) with the Levene's test (for $\Sigma$ ) and Mann-Whitney U-test (for $\sigma$ ). Also, differences in $\Sigma$ and $\sigma$ between superiorly and inferiorly located abdominal organs were tested (i.e., liver vs. right kidney, spleen vs. left kidney). Since differences were tested in 14 combinations (i.e., LR, CC, AP for four organs, and CC only for both diaphragm domes), we adjusted $p$ values according to the Bonferroni correction. Differences 
were considered to be significant if test outcomes showed a $p$ value $<0.004$ (i.e., $0.05 / 14$ ).

We used the Spearman's correlation test (significance level $p<0.05$ ) to investigate the possible correlations in position variations between contralateral organs.

Additionally, to test if right- and left-sided organ position variations in the $\mathrm{CC}$ direction are related to the position variations of the superiorly located right- and left-sided diaphragm dome respectively, we used linear regression analysis (significance level $p<0.05$ ). Both tests were also performed for each individual patient.

All statistical analyses were done using $\mathrm{R}$ version 3.2.1. (R Foundation for Statistical Computing, USA).

\section{Results}

Mean organ position variation was smaller than $1.0 \mathrm{~mm}$ (range: -6.9 to $7.4 \mathrm{~mm}$ ) for the abdominal organs in all orthogonal directions and smaller than $1.8 \mathrm{~mm}$ (range: -4.0 to $7.8 \mathrm{~mm}$ ) for the diaphragm domes in the CC direction. For all organs and across all fractions, ranges of position variations were largest in the $\mathrm{CC}$ direction (varying from
10.6 to $13.0 \mathrm{~mm}$ ) and smallest in the LR direction (varying from 4.1 to $11.1 \mathrm{~mm}$ ) (Fig. 2). Overall, kidney position variations were smaller than position variations of the liver and spleen (Fig. 2). Table 2 presents the values of the group mean, systematic and random error per organ in each direction, mainly showing average systematic error in decreasing order of CC $(3.2 \mathrm{~mm}, \mathrm{SD}=0.3 \mathrm{~mm}), \mathrm{AP}(1.9 \mathrm{~mm}, \mathrm{SD}$ $=0.9 \mathrm{~mm})$, and $\mathrm{LR}(1.7 \mathrm{~mm}, \mathrm{SD}=0.5 \mathrm{~mm})$ direction, and average random error also in decreasing order of $\mathrm{CC}$ (3.0 mm, SD $=0.5 \mathrm{~mm}), \mathrm{AP}(2.1 \mathrm{~mm}, \mathrm{SD}=0.6 \mathrm{~mm})$, and LR $(1.9 \mathrm{~mm}, \mathrm{SD}=0.7 \mathrm{~mm})$ direction. Differences of the systematic error between right- and left-sided organs were insignificant $(p \geq 0.004)$, as were the differences of the random error between right- and left-sided organs $(p \geq 0.004)$ (Additional file 1: Table S1). For superiorly and inferiorly located organs, significant but small differences were found between the liver and the right kidney in the AP direction ( $p=0.002$ for $\Sigma)$, and in the LR direction ( $p$ $=0.000$ for $\sigma$ ). Also, the random error of the spleen and the left kidney was significantly different in the AP direction ( $p$ $=0.001$ ) (Additional file 1: Table S1).
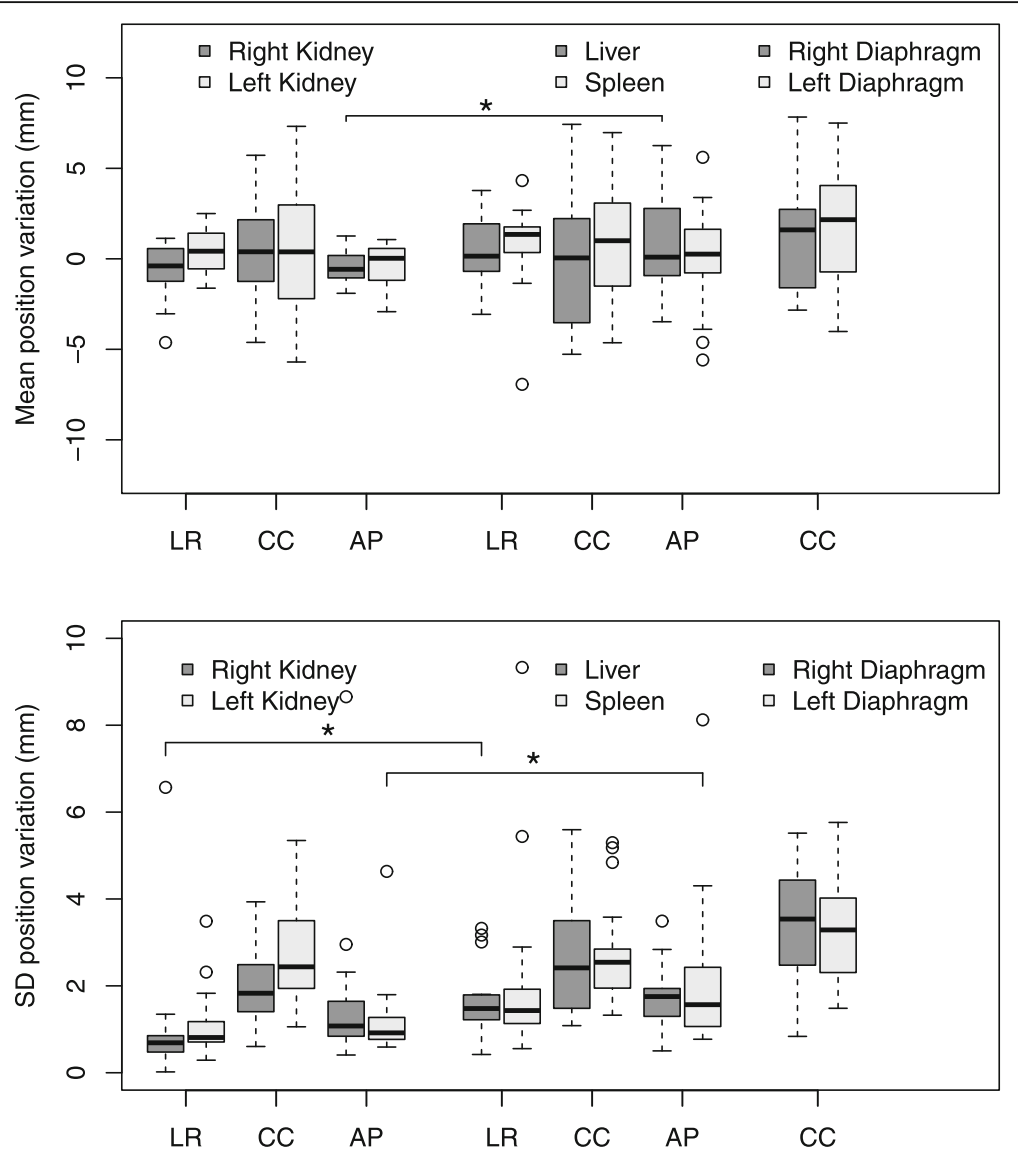

Fig. 2 Boxplots showing the distributions of the individual means (upper panel) and SDs (lower panel) of the interfractional position variations found for right- (light grey) and left-sided (dark grey) organs for all 20 patients. Horizontal bars, boxes, and whiskers represent medians, 50th percentiles (inter quartile range (IQR)), and the highest (lowest) value within 1.5xIQR, respectively. Circles denote outliers. *Significant differences (Bonferroni corrected $p$ $<$ 0.004). Abbreviations: $L R=$ left-right; $C C=$ cranial-caudal; $A P=$ anterior-posterior 
Table 2 The group systematic $(\Sigma)$ and group random errors $(\sigma)$ in $\mathrm{mm}$ in the orthogonal directions for the right kidney, left kidney, liver, and spleen and in CC direction for the diaphragm

\begin{tabular}{|c|c|c|c|c|c|c|c|c|c|c|c|c|c|c|}
\hline \multirow[t]{2}{*}{$(\mathrm{mm})$} & \multicolumn{3}{|c|}{ Right Kidney } & \multicolumn{3}{|c|}{ Left Kidney } & \multicolumn{3}{|c|}{ Liver } & \multicolumn{3}{|c|}{ Spleen } & \multirow{2}{*}{$\frac{\text { Right Diaphragm }}{\mathrm{CC}}$} & \multirow{2}{*}{$\frac{\text { Left Diaphragm }}{\text { CC }}$} \\
\hline & $\mathrm{LR}$ & CC & AP & $\mathrm{LR}$ & CC & AP & $\mathrm{LR}$ & $\mathrm{CC}$ & $\mathrm{AP}$ & $\mathrm{LR}$ & $\mathrm{CC}$ & $\mathrm{AP}$ & & \\
\hline Group mean & -0.6 & 0.7 & -0.4 & 0.4 & 0.4 & -0.4 & 0.4 & -0.1 & 1.0 & 0.8 & 0.8 & 0.0 & 1.2 & 1.8 \\
\hline$\Sigma$ & 1.4 & 2.8 & 0.9 & 1.1 & 3.3 & 1.3 & 2.1 & 3.4 & 2.7 & 2.2 & 3.5 & 2.7 & 3.0 & 3.4 \\
\hline$\sigma$ & 1.6 & 2.2 & 2.4 & 1.3 & 2.9 & 1.4 & 1.8 & 2.8 & 1.9 & 2.8 & 3.0 & 2.7 & 3.6 & 3.4 \\
\hline
\end{tabular}

Abbreviations: $L R$ left-right, $C C$ cranial-caudal, $A P$ anterior-posterior

A moderate and statistically significantly correlation between the position variations of the right and left diaphragm domes was found ( $\rho=0.63, p=0.00$ ) (Fig. 3a). The position variations of the liver and spleen in the LR and CC direction were weakly, but statistically significantly correlated $(\rho=0.23, p=0.02$ and $\rho=0.40, p=$ 0.00 , respectively) (Fig. $3 \mathrm{~b}$ ). Position variations of the right and left kidney were weakly, but statistically significant correlated in the LR and AP directions $(\rho=-0.43$, $p=0.00$ and $\rho=0.23, p=0.01$, respectively) (Fig. 3c). Correlations within each individual patient were similar to the overall group outcomes.

Linear regression analysis showed that right and left diaphragm dome position variations in the CC direction were significantly correlated with position variations of the liver $\left(\mathrm{R}^{2}=0.55, p=0.00\right)$ and spleen $\left(\mathrm{R}^{2}=0.63, p=\right.$ 0.00 ), respectively. In the CC direction, no (strong) correlation was found between right and left diaphragm dome position variations and the position variations of the right $\left(R^{2}=0.003, p=0.60\right)$ and left kidney $\left(R^{2}=0.25\right.$, $p=0.00$ ), respectively (Fig. 4).

\section{Discussion}

In this study, we quantified interfractional position variation of multiple abdominal organs in 20 children during radiotherapy and evaluated if organ position variation is mutually related and location-dependent. We found weak correlations between the position variations of contralateral organs. In the CC direction, right and left diaphragm dome position variations correlated moderately with the position variations of the liver and spleen, respectively. However, correlations between the position variations of the diaphragm domes and those of both kidneys were negligible. Furthermore, the largest magnitude of organ position variations was observed in the $\mathrm{CC}$ direction, followed by the AP and LR directions. We found that differences between group systematic and random errors of abdominal organs were small and insignificant. This comprehensive analysis of organ position variations at different anatomical locations increases the insight in possible consequences for margin definitions, which has not been reported on for children so far.

Nazmy et al. studied interfractional position variation of the liver and kidneys in 9 children (mean age: 4.1 years, $\mathrm{SD}=1.6$ years) using reference $\mathrm{CT}$ and $\mathrm{CBCT}$ scans [10]. They also found that, in the CC direction, the liver showed more motion than the kidneys. However, their range of observed position variations of the left kidney was smaller than that of the right kidney. In contrary, when we analyzed patients in our cohort of similar age ( $n=6$; range $2.2-5.3$ years) we found slightly
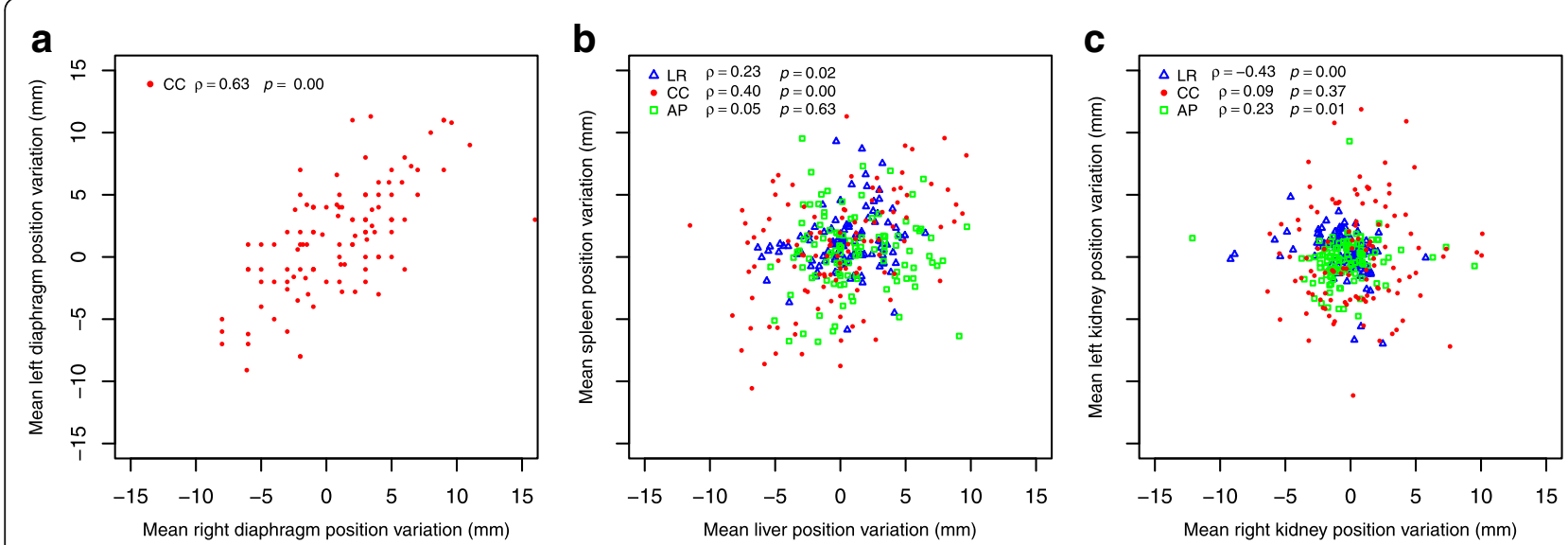

Fig. 3 For all CBCT scans, scatterplots describing relations (Spearman's $\rho$ and $p$-value) between right and left diaphragm position variations in the CC direction only (a) and right- (x-axis) and left-sided (y-axis) organ interfractional position variations separately (b; liver and spleen, $\mathbf{c}$; right and left

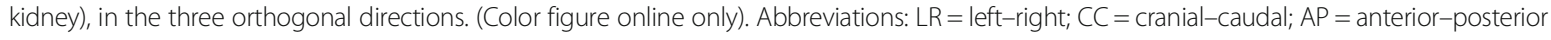



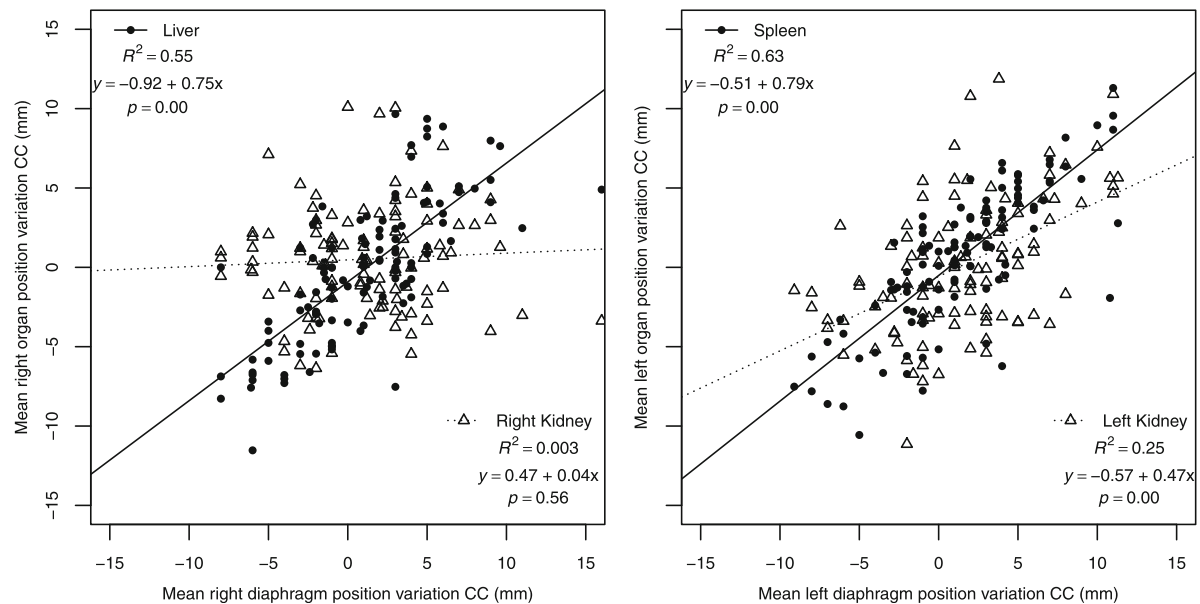

Fig. 4 Scatterplots with regression lines of the linear regression analyses describing relationships for each CBCT between right- and left-sided interfractional organ position variation (y-axis) and diaphragmatic position variation in the CC direction (x-axis). Abbreviations: $C C=c r a n i a l-c a u d a l$

larger position variations of the left kidney compared to the right kidney. Although this comparison involves small sample sizes, a possible explanation might be the different methodology in choosing the point of interest. Nazmy et al. used the upper pole of the kidneys whereby kidney deformations might have been interpreted as translations, resulting in an overestimation of motion. We used the COM as point of interest because it is less sensitive to organ deformations. Although data on organ deformation would provide useful additional information on organ motion characteristics, analyzing organ deformation was outside the scope of the current study.

Our results are comparable to findings of Guerreiro et al. who used a similar methodology as we did [16]. They quantified interfractional position variations of the spleen, liver, and the healthy kidney in patients $(n=15$, mean age: 4 years) with Wilms' tumors. Their ranges of interfractional position variation, and the systematic and random errors were generally somewhat smaller than our results, which could be explained by the fact that their cohort consisted of younger patients (age range 1-8 years). However, when we analyzed patients in our cohort of similar age $(n=10$; range 2.2-7.8 years), the systematic and random errors for all organs and directions in our cohort remained somewhat larger (for $\Sigma$; mean difference $1.0 \mathrm{~mm}, \mathrm{SD}=0.6 \mathrm{~mm}$, for $\sigma$; mean difference $0.7 \mathrm{~mm}, \mathrm{SD}=0.7 \mathrm{~mm}$ ).

Using a $3 \mathrm{DCT}$ as a reference point to estimate interfractional position variation is arguable. The 3DCT represents 'snapshot' of repeatedly changing organ positions during the respiratory cycle [27]. A CBCT captures in 35-60 s several complete respiratory cycles and averages the motion over the observed breathing phases into one blurred 3D image. To investigate the possible effect of respiratory motion differences on the $3 \mathrm{DCT}$ and the CBCTs, we recalculated our measurements using the first CBCT scan as the reference scan instead of the 3DCT. Differences between the respective calculations based on the refCT and the first $\mathrm{CBCT}$ were negligible $(<1 \mathrm{~mm})$. Also, although projection images could enable the quantification of intrafractional motion as well [28], the low dose CBCT protocols that we used for most children [29] unavoidably result in poorer quality of projection images. Therefore, we were not able to distinguish organs on the two-dimensional projection images of these CBCT scans in order to investigate intrafractional motion of the liver, spleen, and kidneys.

The outliers shown in Fig. 2 represent the SD values of the right kidney and spleen position variations of three patients. For one patient, in which the field of view of the CBCT scan was smaller than its refCT, the whole right kidney was visible on the refCT but remained only half visible on the $\mathrm{CBCT}$ scan, and registration was performed using an adjusted sub-volume of the kidney. Additionally, in this patient the distance of the COM of the right kidney to the treatment planning isocentre on the refCT was relatively large $(>10 \mathrm{~mm})$, resulting in a large deviation in organ position variation. For two other patients, the two-step rigid registration for the spleen yielded large rotations (> 15 degrees), resulting in large ranges of position variations. However, a sensitivity analysis, excluding these three cases, did not change our results.

The liver and spleen are contralateral organs that substantially differ in tissue composition and function. However, regarding their position variations, differences were small and position variations of both organs were moderately correlated with the position variations of the diaphragm domes. In contrary, the position variations of both kidneys were smaller and showed weak correlations with the diaphragm dome position variations. This might be due to their more inferior and retroperitoneal location. 
Further, visual inspection showed that the kidneys seem more prone to deformations than the liver and spleen, probably due to their different tissue composition. Therefore, although in the $\mathrm{CC}$ direction only, diaphragm position variations seem to particularly be more representative for position variations of OARs in the upper abdomen than for OAR position variations in the lower abdomen.

The weak to moderate $(\rho<0.4)$, however significant, correlations of position variations between right- and left-sided abdominal organs suggest that organs move only somewhat in similar directions. Therefore, for future online strategies, close located anatomical structures are not recommended as suitable surrogates. However, the overall magnitude of motion is small, and differences of systematic and random errors of the various abdominal organs are small and insignificant, hence negligible. Therefore, regarding margin definitions, there was insufficient evidence of a dependence of organ position variation on anatomical location. Additionally, although differences between abdominal organ position variations were small, overall position variation was largest in the $\mathrm{CC}$ direction and smallest in the LR direction. This suggests that margins should be applied anisotropically rather than isotropically. Note, however, that the diaphragm was measured in the CC direction only.

Knowledge about patient's day-to-day anatomical variation is furthermore valuable when (automating) selecting similar patients from a database of patients' CT scans for, e.g., automating treatment planning or dose reconstruction [30-34], because this provides a lower bound on the achievable precision of selection.

Besides, as recommended by the Paediatric Radiation Oncology Society (PROS), consensus needs to be reached regarding appropriate margin definitions in children [35]. With increasing data, knowledge on organ motion during radiotherapy in children is expanding. However, due to generally small patient numbers and different methodologies in separates studies, definitive statements regarding margin definitions cannot be made yet. Therefore, close collaborations between research groups, and pooling of data might contribute to achieving consensus on margin definitions. A summarized all-encompassing overview of all published data so far, including inter- and intrafractional organ motion, could provide a basis for this. Especially, with more proton and carbon therapy facilities in development, aiming for high-precision radiotherapy and the need for the assessment of the anatomical variations in children, induced by organ motion, becomes even more important.

\section{Conclusions}

No (strong) correlations between interfractional position variations of abdominal organs in children were observed.
Differences of systematic and random errors between abdominal organs were small, suggesting that for margin definitions, there was insufficient evidence of a dependence of organ position variation on anatomical location. From present results, we concluded that diaphragm dome position variations could be more representative for superiorly located abdominal (liver, spleen) organ position variations than for inferiorly located (kidneys) organ position variations.

\section{Additional file}

Additional file 1: Table S1. $p$-values for differences of group systematic errors $(\Sigma)$ and group random errors $(\sigma)$; tested for differences between right- and left-sided organs and superiorly vs. inferiorly located organs in the orthogonal directions. (DOCX $16 \mathrm{~kb}$ )

\section{Abbreviations}

3DCT: 3-Dimensional computed tomography; 4DCT: 4-Dimensional computed tomography; 4DMRI: 4-Dimensional Magnetic Resonance Imaging; AP: Anterior-Posterior; CBCT: Cone Beam Computed Tomography; CC: Cranial-Caudal; COM: Center of Mass; CT: Computed Tomography; LR: Left-Right; OAR: Organ at Risk; PROS: Paediatric Radiation Oncology Society; PRV: Planning Risk Volume; PTV: Planning Target Volume; SD: Standard Deviation

\section{Funding}

This work was supported by the Dutch Cancer Society (KWF Kankerbestrijding) project no. 2016-10113.

\section{Availability of data and materials}

The datasets used and/or analyzed during the current study are available from the corresponding author on reasonable request.

\section{Conflict of interest statement}

Dr. Alderliesten and Dr. Bel are project leaders of several Elekta-sponsored projects outside of this work. Elekta had no involvement in study design, data collection and analysis, or writing of the manuscript.

\section{Authors' contributions}

$\mathrm{SCH}$ has performed the data analysis and carried out writing of the manuscript. IVD, JV, BVB, $T A$ and $A B$ contributed to the critical interpretation of the results. IVD, DTL and $A B$ contributed to design of the manuscript. CRR and $A B$ provided general supervision. All authors participated in the drafting and revising of the manuscript. All authors read and approved the final manuscript.

\section{Ethics approval and consent to participate} Not applicable.

\section{Consent for publication}

Not applicable.

\section{Competing interests}

Dr. Alderliesten and Dr. Bel are project leaders of several Elekta-sponsored projects outside of this work. Elekta had no involvement in study design, data collection and analysis, or writing of the manuscript. The authors declare that they have no competing interests.

\section{Publisher's Note}

Springer Nature remains neutral with regard to jurisdictional claims in published maps and institutional affiliations. 
Received: 30 May 2018 Accepted: 20 August 2018

Published online: 12 September 2018

\section{References}

1. Curry HL, Parkes SE, Powell JE, Mann JR. Caring for survivors of childhood cancers: the size of the problem. Eur J Cancer. 2006:42:501-8.

2. Bolling T, Konemann S, Ernst I, Willich N. Late effects of thoracic irradiation in children. Strahlenther Onkol. 2008;184:289-95.

3. Bolling T, Willich N, Ernst I. Late effects of abdominal irradiation in children: a review of the literature. Anticancer Res. 2010;30:227-31.

4. Selo N, Bolling T, Ernst I, Pape H, Martini C, Rube C, et al. Acute toxicity profile of radiotherapy in 690 children and adolescents: RiSK data. Radiother Oncol. 2010:97:119-26.

5. Claude L, Laprie A. Which dose constraints on which critical organs in paediatric radiation therapy? Cancer Radiother. 2015:19:484-8.

6. Prescribing, recording and reporting photon beam therapy (supplement to ICRU report 50). ICRU report 62: International Commission on Radiation Units and Measurements; 1999.

7. Huijskens SC, van Dijk IW, de Jong R, Visser J, Fajardo RD, Ronckers CM, et al. Quantification of renal and diaphragmatic interfractional motion in pediatric imageguided radiation therapy: a multicenter study. Radiother Oncol. 2015;117:425-31.

8. Huijskens SC, van Dijk IW, Visser J, Rasch CR, Alderliesten T, Bel A. Magnitude and variability of respiratory-induced diaphragm motion in children during image-guided radiotherapy. Radiother Oncol. 2017;123:263-9.

9. Kannan S, Teo BK, Solberg T, Hill-Kayser C. Organ motion in pediatric highrisk neuroblastoma patients using four-dimensional computed tomography. J Appl Clin Med Phys. 2017;18:107-14.

10. Nazmy MS, Khafaga Y, Mousa A, Khalil E. Cone beam CT for organs motion evaluation in pediatric abdominal neuroblastoma. Radiother Oncol. 2012;102:388-92.

11. Pai Panandiker AS, Sharma S, Naik MH, Wu S, Hua C, Beltran C, et al. Novel assessment of renal motion in children as measured via four-dimensional computed tomography. Int J Radiat Oncol Biol Phys. 2012;82:1771-6.

12. Uh J, Krasin MJ, Li Y, Li X, Tinkle C, Lucas JT Jr, et al. Quantification of pediatric abdominal organ motion with a 4-dimensional magnetic resonance imaging method. Int J Radiat Oncol Biol Phys. 2017;99:227-37.

13. Demoor-Goldschmidt C, Chiavassa S, Josset S, Mahe MA, Supiot S. Respiratory-gated bilateral pulmonary radiotherapy for Ewing's sarcoma and nephroblastoma in children and young adults: Dosimetric and clinical feasibility studies. Cancer Radiother. 2017;21:124-9.

14. van Dijk IW, Huijskens SC, de Jong R, Visser J, Fajardo RD, Rasch CR, et al. Interfractional renal and diaphragmatic position variation during radiotherapy in children and adults: is there a difference? Acta Oncol. 2017:1-7.

15. van Herk M. Errors and margins in radiotherapy. Semin Radiat Oncol 2004; 14:52-64.

16. Guerreiro F, Seravalli E, Janssens $G O$, van de Ven $C P$, van den Heuvel-Eibrink MM, Raaymakers BW. Intra- and inter-fraction uncertainties during IGRT for Wilms' tumor. Acta Oncol 2018:1-9.

17. Panandiker AS, Winchell A, Loeffler R, Song R, Rolen M, Hillenbrand C. 4DMRI provides more accurate renal motion estimation in IMRT in young children. Practical radiation oncology. 2013;3:S1

18. Beltran C, Pai Panandiker AS, Krasin MJ, Merchant TE. Daily image-guided localization for neuroblastoma. J Appl Clin Med Phys. 2010;11:3388.

19. Yang J, Cai J, Wang H, Chang Z, Czito BG, Bashir MR, et al. Is diaphragm motion a good surrogate for liver tumor motion? Int J Radiat Oncol Biol Phys. 2014;90:952-8.

20. Xi M, Liu MZ, Li QQ, Cai L, Zhang L, Hu YH. Analysis of abdominal organ motion using four-dimensional CT. Ai Zheng. 2009;28:989-93.

21. Cervino LI, Jiang Y, Sandhu A, Jiang SB. Tumor motion prediction with the diaphragm as a surrogate: a feasibility study. Phys Med Biol. 2010;55:N221-9.

22. Dawson LA, Eccles C, Bissonnette JP, Brock KK. Accuracy of daily image guidance for hypofractionated liver radiotherapy with active breathing control. Int J Radiat Oncol Biol Phys. 2005;62:1247-52.

23. Siva S, Pham D, Gill S, Bressel M, Dang K, Devereux T, et al. An analysis of respiratory induced kidney motion on four-dimensional computed tomography and its implications for stereotactic kidney radiotherapy. Radiat Oncol. 2013;8:248.

24. Lens $E$, van der Horst A, Versteijne $E$, Bel A, van Tienhoven $G$. Considerable pancreatic tumor motion during breath-holding. Acta Oncol. 2016;55:1360-8.
25. Stevens CW, Munden RF, Forster KM, Kelly JF, Liao Z, Starkschall G, et al. Respiratory-driven lung tumor motion is independent of tumor size, tumor location, and pulmonary function. Int J Radiat Oncol Biol Phys. 2001;51:62-8.

26. Roche A, Malandain G, Pennec X, Ayache N. The correlation ratio as a new similarity measure for multimodal image registration. In: Wells WM, Colchester A, Delp S, editors. Medical Image Computing and ComputerAssisted Intervention - MICCAI'98: First International Conference Cambridge, MA, USA, October 11-13, 1998 Proceedings. Berlin, Heidelberg: Springer Berlin Heidelberg; 1998. p. 1115-24.

27. Balter JM, Ten Haken RK, Lawrence TS, Lam KL, Robertson JM. Uncertainties in CT-based radiation therapy treatment planning associated with patient breathing. Int J Radiat Oncol Biol Phys. 1996;36:167-74.

28. Zijp L, Sonke J, van Herk M. Extraction of the respiratory signal from sequential thorax cone-beam X-ray images. Conf Use Comput Radiat Ther (ICCR). 2004:507-9.

29. de Jong RLE, van Herk M, Alderliesten T, Kamphuis M, DF R, Bel A, van Wieringen N. Optimizing cone-beam $C T$ presets for children to reduce imaging dose illustrated with craniospinal axis. In: proceedings of ESTRO 33: Radiother Oncol; 2014. p. S109-S10.

30. Deshpande RR, DeMarco J, Sayre JW, Liu BJ. Knowledge-driven decision support for assessing dose distributions in radiation therapy of head and neck cancer. Int J Comput Assist Radiol Surg. 2016;11:2071-83.

31. Ng A, Nguyen TN, Moseley JL, Hodgson DC, Sharpe MB, Brock KK. Reconstruction of 3D lung models from 2D planning data sets for Hodgkin's lymphoma patients using combined deformable image registration and navigator channels. Med Phys. 2010;37:1017-28.

32. Wang Z, van Dijk I, Wiersma J, Ronckers CM, Oldenburger F, Balgobind BV, et al. Are age and gender suitable matching criteria in organ dose reconstruction using surrogate childhood cancer patients' CT scans? Med Phys. 2018;

33. Schmidt M, Lo JY, Grzetic S, Lutzky C, Brizel DM, Das SK. Semiautomated head-and-neck IMRT planning using dose warping and scaling to robustly adapt plans in a knowledge database containing potentially suboptimal plans. Med Phys. 2015;42:4428-34.

34. Virgolin M, van Dijk I, Wiersma J, Ronckers CM, Witteveen C, Bel A, et al. On the feasibility of automatically selecting similar patients in highly individualized radiotherapy dose reconstruction for historic data of pediatric cancer survivors. Med Phys. 2018;45:1504-17.

35. Kortmann RD, Freeman C, Marcus K, Claude L, Dieckmann K, Halperin E, et al. Paediatric radiation oncology in the care of childhood cancer: a position paper by the international Paediatric radiation oncology society (PROS). Radiother Oncol. 2016;119:357-60.

Ready to submit your research? Choose BMC and benefit from:

- fast, convenient online submission

- thorough peer review by experienced researchers in your field

- rapid publication on acceptance

- support for research data, including large and complex data types

- gold Open Access which fosters wider collaboration and increased citations

- maximum visibility for your research: over $100 \mathrm{M}$ website views per year

At $\mathrm{BMC}$, research is always in progress.

Learn more biomedcentral.com/submissions 